\title{
Revealing the binary origin of Type Ic superluminous supernovae through nebular hydrogen emission
}

\author{
Takashi J. Moriya ${ }^{1}$, Zheng-Wei Liu ${ }^{1}$, Jonathan Mackey ${ }^{2}$, Ting-Wan Chen $^{1}$, and Norbert Langer ${ }^{1}$ \\ 1 Argelander Institute for Astronomy, University of Bonn, Auf dem Hügel 71, 53121 Bonn, Germany \\ e-mail: moriyatk@astro.uni-bonn.de \\ 2 I. Physikalisches Institut, Universität zu Köln, Zülpicher Straße 77, 50937 Köln, Germany \\ Received 6 October 2015 / Accepted 26 October 2015
}

\section{ABSTRACT}

\begin{abstract}
We propose that nebular $\mathrm{H} \alpha$ emission, as detected in the Type Ic superluminous supernova iPTF13ehe, stems from matter that is stripped from a companion star when the supernova ejecta collide with it. The temporal evolution, the line broadening, and the overall blueshift of the emission are consistent with this interpretation. We scale the nebular H $\alpha$ luminosity predicted for Type Ia supernovae in single-degenerate systems to derive the stripped mass required to explain the H $\alpha$ luminosity of iPTF13ehe. We find a stripped mass of 0.1-0.9 solar masses, assuming that the supernova luminosity is powered by radioactivity or magnetar spin down. Because a central heating source is required to excite the $\mathrm{H} \alpha$ emission, an interaction-powered model is not favored for iPTF13ehe if the $\mathrm{H} \alpha$ emission is from stripped matter. We derive a companion mass of more than 20 solar masses and a binary separation of less than about 20 companion radii based on the stripping efficiency during the collision, indicating that the supernova progenitor and the companion formed a massive close binary system. If Type Ic superluminous supernovae generally occur in massive close binary systems, the early brightening observed previously in several Type Ic superluminous supernovae may also be due to the collision with a close companion. Observations of nebular hydrogen emission in future Type Ic superluminous supernovae will enable us to test this interpretation.
\end{abstract}

Key words. supernovae: general - supernovae: individual: iPTF13ehe

\section{Introduction}

Superluminous supernovae (SLSNe) are a newly discovered class of supernovae (SNe; see Gal-Yam 2012 for a review), and their origin is still a mystery. In particular, the origin of Type Ic SLSNe that do not show hydrogen features in the early phases (e.g., Quimby et al. 2011) has been actively discussed. Several scenarios for making Type Ic SLSNe bright have been proposed, such as magnetar spin-down (e.g., Kasen \& Bildsten 2010; Dessart et al. 2012; Inserra et al. 2013; Chen et al. 2015; Metzger et al. 2015), large production of ${ }^{56} \mathrm{Ni}$ (e.g., Gal-Yam et al. 2009; Moriya et al. 2010; Kasen et al. 2011; Dessart et al. 2013; Kozyreva et al. 2014; Whalen et al. 2014), and the interaction of SN ejecta with hydrogen-free dense circumstellar media (e.g., Chevalier \& Irwin 2011; Moriya \& Maeda 2012; Ginzburg \& Balberg 2012; Chatzopoulos \& Wheeler 2012a; Baklanov et al. 2015; Sorokina et al. 2015). Revealing the progenitors and the power source of Type Ic SLSNe is essential for better understanding massive star evolution (Langer 2012), as well as for possibly using Type Ic SLSNe as a standardizable candle (Quimby et al. 2013; Inserra \& Smartt 2014).

Type Ic SLSNe were thought to be hydrogen-free; however, Yan et al. (2015, Y15 hereafter) have recently reported detecting nebular $\mathrm{H} \alpha$ emission at about 250 days after the $r$-band light-curve (LC) peak in the slowly declining Type Ic SLSN iPTF13ehe. The LC evolution and spectral properties of iPTF13ehe are similar to those of SN 2007bi (e.g., Gal-Yam et al. 2009), which is suggested to be a pair-instability SN (e.g., Heger \& Woosley 2002). Y15 estimate that about $10 \%$ of Type Ic SLSNe may have nebular $\mathrm{H} \alpha$ emission as observed in iPTF13ehe.

Y15 suggest that the nebular $\mathrm{H} \alpha$ emission in iPTF13ehe results from the interaction between the SN ejecta and a hydrogen- rich circumstellar shell. If the shell is located far from the progenitor, it takes time for the $\mathrm{SN}$ ejecta to reach the hydrogen-rich shell. Thus, the $\mathrm{H} \alpha$ emission is observed in late phases. They propose that the hydrogen-rich shell is formed by the pulsational pair instability (e.g., Woosley et al. 2007) of the progenitor and that the subsequent explosion may have resulted in iPTF13ehe. The confinement of stellar wind by external photoionization may also result in the formation of a massive circumstellar shell (Mackey et al. 2014). Wang et al. (2015) invoke the necessity to simultaneously consider three suggested luminosity sources in $\mathrm{PPTF} 13 \mathrm{ehe}$, i.e., ${ }^{56} \mathrm{Ni}$, magnetars, and interaction.

In this Letter, we present an alternative interpretation for the nebular $\mathrm{H} \alpha$ emission. We suggest that the hydrogen observed in iPTF13ehe originates in the matter stripped from the companion star contaminating the inner low-velocity layers of the SN ejecta. The photosphere is in the outer high-velocity layers soon after explosion, and we do not observe emission from the inner layers in the early phase. In the nebular phase when the inner layers are transparent, emission from the contaminated inner layers becomes observable. It has been argued that nebular $\mathrm{H} \alpha$ emission from the stripped matter should be detectable in Type Ia SNe that originate in single-degenerate progenitor systems, although this has not yet been observed (Mattila et al. 2005; Leonard 2007; Shappee et al. 2013; Lundqvist et al. 2013, 2015; Maeda et al. 2014). The stripped mass in Type Ia SNe is estimated to be less than $\sim 10^{-3} M_{\odot}$. We apply the same idea to the case of iPTF13ehe and argue that iPTF13ehe may be the first $\mathrm{SN}$ for which the emission of stripped matter from a companion star is detected.

\section{Stripped mass from the companion}

In this section, we estimate the mass that is required to be stripped from the companion star during the collision in order to explain the hydrogen emission observed in iPTF13ehe. 


\subsection{Scaling relation}

Mattila et al. (2005) performed nebular spectral modeling of Type Ia SNe assuming that the inner layers of the ejecta of the W7 model (Nomoto et al. 1984) are contaminated by solarmetallicity matter stripped from the companion. They predict that the nebular $\mathrm{H} \alpha$ luminosity from the stripped matter is $\simeq 5 \times 10^{36} \mathrm{erg} \mathrm{s}^{-1}$ at 380 days after the explosion for a stripped mass of $M_{\mathrm{st}}=0.05 M_{\odot}$. We scale this result to estimate the stripped mass in iPTF13ehe.

We assume that the $\mathrm{H} \alpha$ emission is mainly due to the nonthermal excitation of hydrogen caused by $\gamma$-rays from the nuclear decay of ${ }^{56} \mathrm{Co} \rightarrow{ }^{56} \mathrm{Fe}$ in the nebular phase (e.g., Mattila et al. 2005). The inner layers of the $\mathrm{W} 7$ model at 380 days are optically thin to $\gamma$-rays. Thus, the $\mathrm{H} \alpha$ luminosity $\left(L_{\mathrm{H} \alpha}\right)$ is proportional to $\tau_{\mathrm{ej}, \gamma} L_{\gamma}$, where $\tau_{\mathrm{ej}, \gamma}$ is the $\gamma$-ray optical depth in the ejecta and $L_{\gamma}$ is the $\gamma$-ray luminosity (cf. Kozma \& Fransson 1992). The $\gamma$-ray optical depth $\tau_{\mathrm{ej}, \gamma}$ is proportional to $\rho_{\mathrm{ej}} R_{\mathrm{ej}}$ where $\rho_{\mathrm{ej}}$ is the ejecta density, and $R_{\mathrm{ej}}=\left(2 E_{\mathrm{ej}} / M_{\mathrm{ej}}\right)^{1 / 2} t$ is the characteristic length scale in the ejecta. Because the inner layers of SN ejecta have an almost-constant density profile (e.g., Kasen 2010), we assume $\rho_{\mathrm{ej}} \propto M_{\mathrm{ej}}^{5 / 2} E_{\mathrm{ej}}^{-3 / 2} t^{-3}$. Then, we obtain $\tau_{\mathrm{ej}, \gamma} \propto \rho_{\mathrm{ej}} R_{\mathrm{ej}} \propto M_{\mathrm{ej}}^{2} E_{\mathrm{ej}}^{-1} t^{-2}$. The late-phase $\gamma$-ray luminosity is $L_{\gamma} \propto M_{56 \mathrm{Ni}} \exp \left(-t / \tau_{56} \mathrm{Co}\right)$ where $M_{56 \mathrm{Ni}}$ is the initial ${ }^{56} \mathrm{Ni}$ mass, and $\tau_{56} \mathrm{Co}=111$ days is the ${ }^{56} \mathrm{Co}$ decay timescale. Finally, the $\mathrm{H} \alpha$ luminosity is proportional to the stripped mass $M_{\mathrm{st}}$. Thus, we expect that the $\mathrm{H} \alpha$ luminosity scales as $L_{\mathrm{H} \alpha} \propto$ $M_{\mathrm{st}} M_{\mathrm{ej}}^{2} E_{\mathrm{ej}}^{-1} M_{56 \mathrm{Ni}} t^{-2} \exp \left(-t / \tau^{56} \mathrm{Co}\right)$.

Using the W7 parameters $\left(M_{\mathrm{ej}}=1.4 M_{\odot}, E_{\mathrm{ej}}=1.3 \mathrm{~B}\right.$, and $\left.M_{56 \mathrm{Ni}}=0.6 M_{\odot}\right)$ and $L_{\mathrm{H} \alpha} \simeq 5 \times 10^{36} \mathrm{erg} \mathrm{s}^{-1}$ at $t=380$ days with $M_{\text {st }}=0.05 M_{\odot}$ (Mattila et al. 2005), we obtain the scaling relation

$$
\begin{aligned}
L_{\mathrm{H} \alpha} \simeq & 5 \times 10^{44}\left(\frac{M_{\mathrm{st}}}{M_{\odot}}\right)\left(\frac{M_{\mathrm{ej}}}{M_{\odot}}\right)^{2}\left(\frac{E_{\mathrm{ej}}}{\mathrm{B}}\right)^{-1}\left(\frac{M^{56} \mathrm{Ni}}{M_{\odot}}\right) \\
& \times\left(\frac{t}{\text { day }}\right)^{-2} \exp \left(-\frac{t}{111 \text { days }}\right) \mathrm{erg} \mathrm{s}^{-1} .
\end{aligned}
$$

We apply this formula to iPTF13ehe in the next section. Its validity and uncertainties for this application are discussed in Sect. 2.3 .

\subsection{The stripped mass in iPTF13ehe}

iPTF13ehe is observed to have $L_{\mathrm{H} \alpha} \simeq 2 \times 10^{41} \mathrm{erg} \mathrm{s}^{-1}$ at $\sim 250$ days after the LC peak (Y15). The rise time is uncertain, so we adopt $\sim 110$ days based on the polynomial fit to the LC (Y15). Given the redshift of iPTF13ehe $(0.3434$, Y15), we estimate that the above $\mathrm{H} \alpha$ luminosity is observed at about 270 days after the explosion in the rest frame. Y15 find that the $\mathrm{H} \alpha$ luminosity decreases by roughly $20 \%$ from 270 days to 290 days in the rest frame. Our interpretation that the stripped matter provides the $\mathrm{H} \alpha$ luminosity predicts about $28 \%$ luminosity reduction during this period (Eq. (1)). This is consistent with the observation.

If iPTF13ehe is powered by ${ }^{56} \mathrm{Ni}$, the required ${ }^{56} \mathrm{Ni}$ mass to obtain the peak luminosity is about $15 M_{\odot}$ (Y15). If we assume that iPTF13ehe is a pair-instability $\mathrm{SN}$, the expected ejecta mass and explosion energy are roughly $110 M_{\odot}$ and $60 \mathrm{~B}$, respectively (e.g., Heger \& Woosley 2002). Substituting these properties for Eq. (1), we obtain $M_{\text {st }} \simeq 0.1 M_{\odot}$. If the large ${ }^{56} \mathrm{Ni}$ production is due to a core-collapse SN (e.g., Umeda \& Nomoto 2008), the ejecta mass can be less than what we expect from the pairinstability SN (e.g., Moriya et al. 2010). A high explosion energy is still required to have the large ${ }^{56} \mathrm{Ni}$ production. If we adopt $M_{\mathrm{ej}}=70 M_{\odot}$ and $E_{\mathrm{ej}}=60 \mathrm{~B}$, we obtain $M_{\mathrm{st}}=0.3 M_{\odot}$.
Table 1. Estimated stripped mass $M_{\mathrm{st}}$ for different progenitor models.

\begin{tabular}{lcccc}
\hline \hline Model & $M_{\mathrm{ej}} / M_{\odot}$ & $E_{\mathrm{ej}} / \mathrm{B}$ & $M_{56 \mathrm{Ni}} / M_{\odot}$ & $M_{\mathrm{st}} / M_{\odot}$ \\
\hline PISN & 110 & 60 & 15 & 0.1 \\
Core-collapse SN & 70 & 60 & 15 & 0.3 \\
Magnetar & 5 & 1 & $(15)$ & 0.9 \\
Wang et al. (2015) & 35 & 40 & $(15)$ & 0.7 \\
Interaction & 5 & 10 & 1 & 140 \\
\hline Type Ia (ref. model) & 1.4 & 1.3 & 0.6 & 0.05 \\
\hline
\end{tabular}

Notes. Values in parentheses for $M_{56 \mathrm{Ni}}$ denote an energy input consistent with that provided by this mass of ${ }^{56} \mathrm{Ni}$, even though this energy may be injected by a central engine.

Even if iPTF13ehe is powered by a magnetar, a $\gamma$-ray deposition from the magnetar similar to the one in the ${ }^{56} \mathrm{Ni}$-powered model is required to explain its slowly declining LC. Thus, we estimate the stripped mass by assuming $M_{56} \mathrm{Ni}=15 M_{\odot}$ in Eq. (1) even in the magnetar model. The main difference between the magnetar-powere and ${ }^{56} \mathrm{Ni}$-powered models is in the $\mathrm{SN}$ ejecta mass and energy. If we take typical ejecta mass $\left(5 M_{\odot}\right)$ and energy (1 B) estimated for magnetar-driven SLSNe (e.g., Inserra et al. 2013; Nicholl et al. 2015a), we obtain $M_{\text {st }} \simeq 0.9 M_{\odot}$. However, the magnetar model for iPTF13ehe by Wang et al. (2015) requires $M_{\mathrm{ej}}=35 M_{\odot}$ and $E_{\mathrm{ej}}=40 \mathrm{~B}$. In this case, the stripped mass is $M_{\mathrm{st}} \simeq 0.7 M_{\odot}$.

If the interaction between the SN ejecta and a hydrogen-free dense circumstellar medium is the major luminosity source of iPTF13ehe, the SN explosion itself can be normal. For instance, the Type Ic SLSN 2010gx had a spectrum that is similar to those observed in broad-line Type Ic SNe (Pastorello et al. 2010). If we adopt typical broad-line Type Ic SN properties $\left(E_{\mathrm{ej}}=10 \mathrm{~B}\right.$, $M_{\mathrm{ej}}=5 M_{\odot}$, and $M^{56} \mathrm{Ni}=1 M_{\odot}$, e.g., Taddia et al. 2015), we obtain $M_{\text {st }} \simeq 140 M_{\odot}$, which is unrealistically high. If we assume a higher ejecta mass, for example $M_{\mathrm{ej}}=20 M_{\odot}$, we obtain a lower stripped mass, $8 M_{\odot}$. A significant central heating source is necessary to excite hydrogen to explain the nebular $\mathrm{H} \alpha$ emission from the stripped mass. We note that a reverse shock created by the interaction, which could propagate into the inner layers to excite hydrogen, does not move rapidly inward because the interaction forms a radiative, dense, cool shell (e.g., Sorokina et al. 2015). Table 1 summarizes the stripped masses estimated in this section.

\subsection{Consistency check and uncertainties}

In Eq. (1), it is assumed that the inner layers of the SN ejecta are optically thin to $\gamma$-rays as in the Type Ia SN model of Mattila et al. (2005) at $t=380$ days. Assuming a $\gamma$-ray opacity of $0.03 \mathrm{~cm}^{2} \mathrm{~g}^{-1}$ (Axelrod 1980), the Type Ia SN model has $\tau_{\mathrm{ej}, \gamma} \sim 10^{-2}$. For the models at 270 days in the previous section, we obtain $\tau_{\mathrm{ej}, \gamma} \sim 1$. Thus, the scaling relation is still able to provide a rough estimate for the stripped mass. Because the optical depth is around unity, the scaling relation may slightly underestimate the stripped mass.

Y15 estimate a ${ }^{56} \mathrm{Ni}$ mass of $2.5 M_{\odot}$ in iPTF13ehe based on their nebular spectral modeling. This ${ }^{56} \mathrm{Ni}$ mass is too low to explain the peak luminosity of iPTF13ehe, leading to the suggestion that two or more luminosity sources power iPTF13ehe (Y15 and Wang et al. 2015). However, this ${ }^{56} \mathrm{Ni}$ mass estimate assumes that ${ }^{56} \mathrm{Ni}$ is irrelevant to the $\mathrm{H} \alpha$ emission. If the $\mathrm{H} \alpha$ emission is powered by radioactive decay, the mass estimate of ${ }^{56} \mathrm{Ni}$ increases. The estimated ${ }^{56} \mathrm{Ni}$ mass is also uncertain because of the uncertainties in the spectral modeling itself (Y15). Thus, the ${ }^{56} \mathrm{Ni}$-powered model with $15 M_{\odot}$ of ${ }^{56} \mathrm{Ni}$ is not ruled out if the $\mathrm{H} \alpha$ emission is emitted by the stripped matter. 


\section{Constraint on the progenitor binary system}

Liu et al. (2015b) have recently studied the collision between $\mathrm{SN}$ ejecta and its companion star in core-collapse $\mathrm{SNe}$ with different separations, companion masses, ejecta masses, and explosion energies (see also Hirai et al. 2014). They investigate how the stripping fraction $f \equiv M_{\text {st }} / M_{2}$ (where $M_{2}$ is the companion mass before the collision) is affected by these parameters. Liu et al. (2015b) only investigate the collision with the companion stars of 0.9 and $3.5 M_{\odot}$. To constrain the massive progenitor system of iPTF13ehe, we performed numerical simulations of the collision between the SN ejecta $\left(110 M_{\odot}\right.$ and $60 \mathrm{~B})$ and a massive main-sequence $\operatorname{star}\left(50 M_{\odot}\right.$ with $\left.10 R_{\odot}\right)$ by adopting the same method as in Liu et al. (2015b). The threedimensional smoothed-particle hydrodynamics code Stellar GADGET (Pakmor et al. 2012) is used for the simulations. We ran simulations for two separations, $5 R_{2}$ and $10 R_{2}$, where $R_{2}$ is the companion radius. The Roche-lobe radii for the mass ratios of 0.5 and 1 are 3.1 $R_{2}$ and 2.6 $R_{2}$, respectively (Eggleton 1983).

The numerical simulations reveal stripped masses of $0.3 M_{\odot}$ $\left(f \simeq 6 \times 10^{-3}\right)$ and $0.05 M_{\odot}\left(f \simeq 10^{-3}\right)$ for the separations of $5 R_{2}$ and $10 R_{2}$, respectively. The stripped mass therefore reaches more than $0.1 M_{\odot}$, as required from our estimate, and the binary system with the $50 M_{\odot}$ main-sequence companion is consistent with the $\mathrm{H} \alpha$ luminosity observed in iPTF13ehe. When assuming $f=(6-1) \times 10^{-3}$, the companion mass needs to be around 20-100 $M_{\odot}$ if $M_{\text {st }}=0.1 M_{\odot}$. Because the ejecta mass does not strongly affect the stripping fraction (Liu et al. 2015b), the companion mass is expected to be $50-300 M_{\odot}$ if the ejecta mass is $70 M_{\odot}\left(M_{\text {st }}=0.3 M_{\odot}\right)$.

The stripping fraction is proportional to $E_{\mathrm{ej}}$ (Liu et al. 2015b). The magnetar model in Wang et al. (2015) has a high explosion energy (40 B), and the companion mass needs to be more than about $80 M_{\odot}$ to have $M_{\mathrm{st}} \simeq 0.7 M_{\odot}$. If $E_{\mathrm{ej}} \simeq 1 \mathrm{~B}$, as estimated from the typical magnetar model, the stripping fraction is likely to be $f \sim 10^{-4}$ or less. Then, the companion mass needs to be higher than $\sim 10^{4} M_{\odot}\left(M_{\mathrm{st}}=0.9 M_{\odot}\right)$, which is extremely high.

The stripped mass strongly depends on the separation. As the separation becomes larger, the angular size of the companion star from the SN ejecta becomes smaller. Thus, less energy is ejected in the direction of the companion and the mass stripping becomes less efficient. The above stripping fraction is estimated for separations between $5 R_{2}$ and $10 R_{2}$. As indicated in Liu et al. (2015b), the stripping fraction drops significantly as the separation doubles (see also Hirai et al. 2014). If the separation is more than about $20 R_{2}$, the required companion mass is likely to become more than $10^{3} M_{\odot}$ in most of the models.

\section{Discussion}

\subsection{Ho emission features}

So far, we have focused on the observed $\mathrm{H} \alpha$ luminosity of iPTF13ehe. Here we discuss other $\mathrm{H} \alpha$ features of iPTF13ehe in the context of our stripping scenario.

The $\mathrm{H} \alpha$ emission in iPTF13ehe is observed to have a line broadening of $\simeq 4000 \mathrm{~km} \mathrm{~s}^{-1}$ and an overall line shift of several $100 \mathrm{~km} \mathrm{~s}^{-1}$ (Y15). Because the stripped matter remains within the inner layers of the SN ejecta, it is expected to emit at relatively low velocities $\left(\lesssim 1000 \mathrm{~km} \mathrm{~s}^{-1}\right.$ in the case of Type Ia SNe, e.g., Liu et al. 2012). If the explosion energy is higher, stripped matter can end up with higher velocities, and we expect to observe broader emission lines, as seen in iPTF13ehe. In Fig. 1, we show the velocity distribution of the stripped matter in our impact simulation with a separation $5 R_{2}$. The typical velocity of

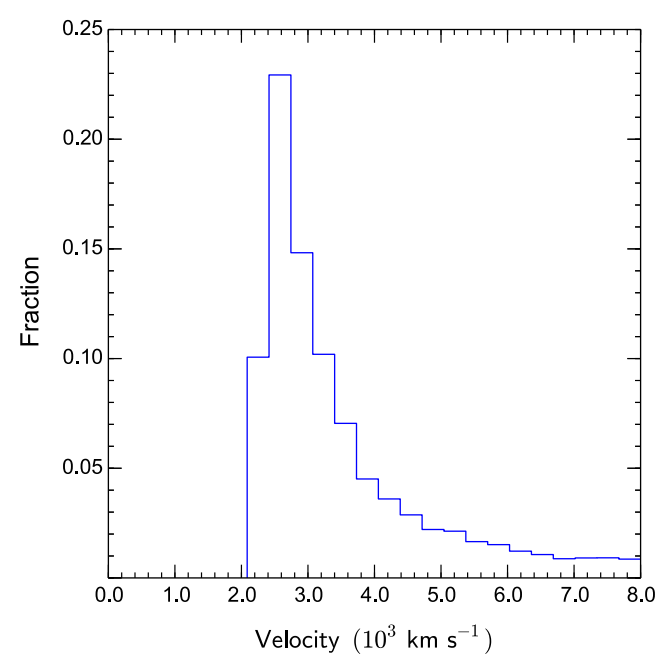

Fig. 1. Final velocity distribution of the stripped matter from the mainsequence companion star in our numerical simulation with a separation of $5 R_{2}$.

the stripped matter is around $3000 \mathrm{~km} \mathrm{~s}^{-1}$, while the typical velocity of the ejecta is around $8000 \mathrm{~km} \mathrm{~s}^{-1}$. The velocities of the stripped matter are actually lower than the typical ejecta velocity, and they are close to the line broadening observed in iPTF13ehe. If the companion star is located between the progenitor and the observer at the time of the explosion, the collision occurs in the ejecta moving toward the observer. Thus, we expect that the stripped matter has an overall velocity toward the observer and that an observer measures blueshifted $\mathrm{H} \alpha$ emission. If the companion is in the opposite direction, the emission is likely to have an overall redshift. Thus, for emission from the stripped matter, we expect to eventually observe an overall redshift in other Type Ic SLSNe. Detailed modeling of the nebular spectra based on impact simulations similar to Liu et al. (2015b) is required to investigate the expected emission properties further.

\subsection{Early brightening observed in Type Ic SLSNe}

Some Type Ic SLSNe are known to have an early brightening before the main LC rise (Nicholl et al. 2015b; Leloudas et al. 2012). This early brightening has been related to, for example, circumstellar interaction (Moriya \& Maeda 2012) and magnetar spin-down (Kasen et al. 2015). However, if Type Ic SLSNe originate in binary systems, this early emission may be related to the collision between the SN ejecta and the companion (Kasen 2010). We expect an early luminosity of $\sim(1-5) \times 10^{43} \mathrm{erg} \mathrm{s}^{-1}$ within ten days after the explosion from the analytic formula of Kasen (2010), assuming $M_{\mathrm{ej}}=110 M_{\odot}$, an ejecta velocity of $13000 \mathrm{~km} \mathrm{~s}^{-1}$ (Y15), and a separation of $10^{13} \mathrm{~cm}$. This is consistent with the early luminosity observed in Type Ic SLSNe (Leloudas et al. 2012; Nicholl et al. 2015b). The luminosity estimate is not sensitive to $M_{\mathrm{ej}}$, and we expect similar luminosity in the magnetar model (Kasen 2010). Because the general likelihood of observing early brightening by the companion star in Type Ic SNe is estimated to be low (Moriya et al. 2015), the frequent observations of an early brightening in Type Ic SLSNe may also imply that they tend to occur in close binary systems. Early brightening by impact onto a companion star strongly depends on the direction to the observer, while $\mathrm{H} \alpha$ emission from stripped matter can be observed from all directions (e.g., Kasen 2010; Liu et al. 2015a). Thus, $\mathrm{H} \alpha$ emission may be observed more frequently. For example, if we assume a companion viewing angle of about ten degrees, as is nearly the case for $5 R_{2}$, about $10 \%$ of randomly oriented observers are in a preferred direction for finding the early brightening (Kasen 2010). 


\subsection{The progenitor of iPTF13ehe and its binary evolution}

It has been suggested that binary evolution is important for SLSN progenitors (e.g., Justham et al. 2014). That we find a possible signature of the companion star in Type Ic SLSN iPTF13ehe suggests that binary evolution can indeed play a key role in their progenitors. For example, binarity can help progenitors to have a large angular momentum through tidal interactions (e.g., de Mink et al. 2009; Song et al. 2015) and mass transfer (e.g., de Mink et al. 2013). Rotationally-induced mixing may reduce the initial mass needed to obtain PISNe (e.g., Yoon et al. 2012; Chatzopoulos \& Wheeler 2012b; Yusof et al. 2013). Rapid rotation is also essential in the magnetar-powered model because its energy source is rotation.

Y15 estimate that about $10 \%$ of Type Ic SLSNe may have nebular $\mathrm{H} \alpha$ emission. This may indicate that insufficient mass is stripped from the companion to have detectable nebular $\mathrm{H} \alpha$ emission in about $90 \%$ of Type Ic SLSNe currently observed. For example, if the typical separation is larger than about $20 R_{2}$, we do not expect sufficient stripping to be able to observe the emission, as discussed. Alternatively, many progenitors may be effectively single stars. This does not mean that they are single stars from the beginning. A stellar merger leads to an apparent single star (cf. Justham et al. 2014) and to the increase in its rotational velocity (cf. de Mink et al. 2013).

\section{Conclusions}

We have suggested that the $\mathrm{H} \alpha$ emission observed during the nebular phases of Type Ic SLSN iPTF13ehe originates in matter that is stripped off a hydrogen-rich companion star of the SN progenitor. Matter near the surface of the companion star is stripped when the SN ejecta collide with the companion, and the hydrogen-rich stripped matter contaminates the inner lowvelocity layers of the SN ejecta. Emission from the contaminated ejecta can only be observed during the nebular phases when the inner layers are transparent. The temporal evolution, the line broadening, and the overall blueshift of the observed $\mathrm{H} \alpha$ emission in iPTF13ehe are consistent with emission from stripped matter. By scaling the predicted $\mathrm{H} \alpha$ luminosity based on the W7 model of Type Ia SNe, we estimated a stripped mass in iPTF13ehe between 0.1 and $0.9 M_{\odot}$ (Table 1). We do not expect nebular $\mathrm{H} \alpha$ emission from the stripped matter if iPTF13ehe is powered by the interaction between the $\mathrm{SN}$ ejecta and a dense circumstellar medium because a central heating source is required to excite hydrogen located at the inner low-velocity layers of the SN ejecta.

We performed numerical simulations of the mass stripping using the same technique as in Liu et al. (2015b). We assumed $M_{\mathrm{ej}}=110 M_{\odot}$ and $E_{\mathrm{ej}}=60 \mathrm{~B}$ and put a main-sequence companion star with $50 M_{\odot}$ and $10 R_{\odot}$ at a separation of $5 R_{2}$ and $10 R_{2}$. We find stripped masses of $0.3 M_{\odot}\left(5 R_{2}\right)$ and $0.05 M_{\odot}\left(10 R_{2}\right)$. Our mass stripping efficiency implies that the companion mass is higher than about $20 M_{\odot}\left({ }^{56} \mathrm{Ni}\right.$-powered models $)$ or more than about $80 M_{\odot}$ (magnetar spin-down models). Our results imply that the progenitor evolved in a massive binary system and that stellar binarity may play a critical role in the evolution of Type Ic SLSN progenitors. If Type Ic SLSN progenitors are typically in close binary systems, the early brightening sometimes observed in them may also be related to the collision.

If our interpretation of the origin of the $\mathrm{H} \alpha$ emission is correct, we expect some diversity in the $\mathrm{H} \alpha$ emission feature. Especially, the overall line shift, which is a blue shift in the case of iPTF13ehe, is likely to be affected by the viewing angle. If the companion star is located behind the $\mathrm{SN}$ progenitor at the time of explosion, an overall redshift of the $\mathrm{H} \alpha$ emission may be observed. It will also be interesting to obtain the $\mathrm{H} \alpha$ detection rate separately in slowly declining and fast-declining Type Ic SLSNe in future SLSN studies. A large number of observations of late-phase emission from Type Ic SLSNe can test our interpretation and reveal the origin and nature of mysterious Type Ic SLSNe.

Acknowledgements. T.J.M. is supported by Japan Society for the Promotion of Science Postdoctoral Fellowships for Research Abroad (26 -51). J.M. acknowledges support from the Deutsche Forschungsgemeinschaft priority program 1573, Physics of the Interstellar Medium.

\section{References}

Axelrod, T. S. 1980, Ph.D. Thesis, University of California, Santa Cruz Baklanov, P. V., Sorokina, E. I., \& Blinnikov, S. I. 2015, Astron. Lett., 41, 95 Chatzopoulos, E., \& Wheeler, J. C. 2012a, ApJ, 760, 154

Chatzopoulos, E., \& Wheeler, J. C. 2012b, ApJ, 748, 42

Chen, T.-W., Smartt, S. J., Jerkstrand, A., et al. 2015, MNRAS, 452, 1567

Chevalier, R. A., \& Irwin, C. M. 2011, ApJ, 729, L6

de Mink, S. E., Cantiello, M., Langer, N., et al. 2009, A\&A, 497, 243

de Mink, S. E., Langer, N., Izzard, R. G., et al. 2013, ApJ, 764, 166

Dessart, L., Hillier, D. J., Waldman, R., et al. 2012, MNRAS, 426, L76

Dessart, L., Waldman, R., Livne, E., et al. 2013, MNRAS, 428, 3227

Eggleton, P. P. 1983, ApJ, 268, 368

Gal-Yam, A. 2012, Science, 337, 927

Gal-Yam, A., Mazzali, P., Ofek, E. O., et al. 2009, Nature, 462, 624

Ginzburg, S., \& Balberg, S. 2012, ApJ, 757, 178

Heger, A., \& Woosley, S. E. 2002, ApJ, 567, 532

Hirai, R., Sawai, H., \& Yamada, S. 2014, ApJ, 792, 66

Inserra, C., \& Smartt, S. J. 2014, ApJ, 796, 87

Inserra, C., Smartt, S. J., Jerkstrand, A., et al. 2013, ApJ, 770, 128

Justham, S., Podsiadlowski, P., \& Vink, J. S. 2014, ApJ, 796, 121

Kasen, D. 2010, ApJ, 708, 1025

Kasen, D., \& Bildsten, L. 2010, ApJ, 717, 245

Kasen, D., Woosley, S. E., \& Heger, A. 2011, ApJ, 734, 102

Kasen, D., Metzger, B. D., \& Bildsten, L. 2015, ApJ, submitted [arXiv: 1507.03645]

Kozma, C., \& Fransson, C. 1992, ApJ, 390, 602

Kozyreva, A., Blinnikov S., Langer, N., \& Yoon, S.-C. 2014, A\&A, 565, A70 Langer, N. 2012, ARA\&A, 50, 107

Leloudas, G., Chatzopoulos, E., Dilday, B., et al. 2012, A\&A, 541, A129

Leonard, D. C. 2007, ApJ, 670, 1275

Lundqvist, P., Nyholm, A., Taddia, F., et al. 2015, A\&A, 577, A39

Lundqvist, P., Mattila, S., Sollerman, J., et al. 2013, MNRAS, 435, 329

Liu, Z. W., Pakmor, R., Röpke, F. K., et al. 2012, A\&A, 548, A2

Liu, Z.-W., Moriya, T. J., \& Stancliffe, R. J. 2015a, MNRAS, 454, 1192

Liu, Z.-W., Tauris, T. M., Roepke, F. K., et al. 2015b, A\&A, 584, A11

Mackey, J., Mohamed, S., \& Gvaramadze, V. V., et al. 2014, Nature, 512, 282

Maeda, K., Kutsuna, M., \& Shigeyama, T. 2014, ApJ, 794, 37

Mattila, S., Lundqvist, P., Sollerman, J., et al. 2005, A\&A, 443, 649

Metzger, B. D., Margalit, B., Kasen, D., \& Quataert, E. 2015, MNRAS, submitted [arXiv: 1508.02712]

Moriya, T. J., \& Maeda, K. 2012, ApJ, 756, L22

Moriya, T., Tominaga, N., Tanaka, M., et al. 2010, ApJ, 717, L83

Moriya, T. J., Liu, Z.-W., \& Izzard, R. G. 2015, MNRAS, 450, 3264

Nicholl, M., Smartt, S. J., Jerkstrand, A., et al. 2015a, MNRAS, 452, 3869

Nicholl, M., Smartt, S. J., Jerkstrand, A., et al. 2015b, ApJ, 807, L18

Nomoto, K., Thielemann, F.-K., \& Yokoi, K. 1984, ApJ, 286, 644

Pakmor, R., Edelmann, P., Röpke, F. K., et al. 2012, MNRAS, 424, 222

Pastorello, A., Smartt, S. J., Botticella, M. T., et al. 2010, ApJ, 724, L16

Quimby, R. M., Kulkarni, S. R., Kasliwal, M. M., et al. 2011, Nature, 474, 487

Quimby, R. M., Yuan, F., Akerlof, C., \& Wheeler, J. C. 2013, MNRAS, 431, 912 Shappee, B. J., Stanek, K. Z., Pogge, R. W., et al. 2013, ApJ, 762, L5

Song, H. F., Meynet, G., Maeder, A., Ekstrom, S., \& Eggenberger, P. 2015, A\&A, in press, DOI: 10.1051/0004-6361/201526074

Sorokina, E., Blinnikov, S., Nomoto, K., Quimby, R., \& Tolstov, A. 2015, ApJ, submitted [arXiv: 1510.00834]

Taddia, F., Hofmann, R., Mohr, S., et al. 2015, A\&A, 574, A60

Umeda, H., \& Nomoto, K. 2008, ApJ, 673, 1014

Wang, S. Q., Liu, L. D., Dai, Z. G., Wang, L. J., \& Wu, X. F. 2015, ApJ, submitted [arXiv: 1509.05543]

Whalen, D. J., Smidt, J., Heger, A., et al. 2014, ApJ, 797, 9

Woosley, S. E., Blinnikov, S., \& Heger, A. 2007, Nature, 450, 390

Yan, L., Quimby, R., Ofek, E., et al. 2015, ApJ, submitted [arXiv: 1508. 04420] (Y15)

Yoon, S.-C., Dierks, A., \& Langer, N. 2012, A\&A, 542, A113

Yusof, N., Hirschi, R., Meynet, G., et al. 2013, MNRAS, 433, 1114 